\title{
Appropriation de l'espace public urbain : entre aménagements et vécus quotidiens d'un centre commercial
}

Appropriation of the urban public place: between arrangements and daily real-

life experiences

Aneignung vom öffentlichen städtischen Raum : Einrichtung und alltägliche

Nutzung

Thibaut Besozzi

\section{OpenEdition}

\section{Journals}

Electronic version

URL: http://journals.openedition.org/rge/5209

DOI: $10.4000 /$ rge.5209

ISSN: 2108-6478

\section{Publisher}

Association des géographes de l'Est

Printed version

Date of publication: 30 December 2014

ISSN: 0035-3213

\section{Electronic reference}

Thibaut Besozzi, «Appropriation de l'espace public urbain : entre aménagements et vécus quotidiens d'un centre commercial », Revue Géographique de l'Est [Online], vol. 54 / n³-4 | 2014, Online since 01

February 2015, connection on 10 December 2020. URL : http://journals.openedition.org/rge/5209 ;

DOI : https://doi.org/10.4000/rge.5209

This text was automatically generated on 10 December 2020.

Tous droits réservés 


\section{Appropriation de l'espace public urbain : entre aménagements et vécus quotidiens d'un centre commercial}

Appropriation of the urban public place: between arrangements and daily real-

life experiences

Aneignung vom öffentlichen städtischen Raum : Einrichtung und alltägliche

Nutzung

Thibaut Besozzi

\section{Introduction}

1 Le but de cette contribution est d'apporter des éléments empiriques de réflexion sur la question de l'appropriation de l'espace public urbain, et ce, à partir de l'étude ethnographique d'un centre commercial situé au centre-ville d'une agglomération de 300000 habitants, dans le Nord-Est de la France. Le détournement d'usage (Certeau, 1990) de cet espace économique par un ensemble de personnes âgées soulève la question des mécanismes par lesquels ils s'approprient effectivement les galeries marchandes et celle des tactiques qu'ils emploient pour stabiliser leur groupe de sociabilité et leurs pratiques sociales et spatiales non-conventionnelles.

Nous sommes allés observer la vie quotidienne du Centre Commercial Saint-Cyprien ${ }^{1}$ et ce, en concentrant notre attention sur les "aires de repos $\|^{2}$, bancs publics et autres rambardes qui permettent aux clients de s'immobiliser et de se détendre. Nous y avons alors constaté l'existence d'un phénomène d'appropriation spatiale et de sociabilité qui atteste du détournement d'usage du centre commercial. Cette sociabilité peut être dite "primaire », selon la définition de Caillé (1998); elle est le fait d'individus et de groupes qui sont majoritairement composés de personnes âgées retraitées issues des 
classes défavorisées et relativement en proie à la solitude et à l'ennui. Nous les appellerons les « habitués » et les opposerons aux « clients " ${ }^{3}$ de par l'usage qu'ils font des aires de repos.

3 Nous avons pu remarquer l'existence d'un " groupe-diffus » d'habitués émergeant de ce que Chombart de Lauwe appelle leur «milieu social» (1963). Cela correspond, selon le sociologue urbain «à des rassemblements d'hommes qui ne constituent pas nécessairement un groupe, et pourtant sont liés par des représentations communes, des facilités de communication et certains comportements semblables». Autrement dit, les habitués développent une interconnaissance plus ou moins prononcée au sein de l'espace local qu'ils investissent. Si nous avons pu dénombrer environ 80 personnes constituant le microcosme du Centre, tous ne sont pas présents en même temps ni en rapport direct les uns avec les autres: ils constituent plutôt des petits groupes (de 2 à 8 personnes) qui peuvent interagir entre eux à l'occasion. Les habitués se reconnaissent parce qu'ils se rencontrent régulièrement ici, bien qu'ils n'effectuent que peu ou pas d'achats. En ce sens, il ne convient pas de se représenter le Centre comme un «non-lieu » (Augé, 1992), mais bien de voir au contraire comment il devient un lieu à part entière - chargé d'histoire, de relation et d'identité, selon la définition de Augé (1992) - notamment à partir de la pratique clandestine qu'en font les habitués. L'existence de leur petit monde permet de nuancer les résultats de Chivallon, Marme et Prost, pour qui les centres commerciaux ne sont que des « artefacts de lieu » (1997).

4 Nous proposons une analyse centrée sur les rapports spatiaux que donnent à voir ces personnes. Les acteurs concernés s'approprient les micro-espaces d'immobilité du Centre et détournent silencieusement l'usage et le sens premier du lieu. Ils le transforment en un petit monde commun au sein duquel ils se rencontrent régulièrement sur un mode personnalisé. D'autres normes (utilitaires, marchandes, impersonnelles) président conventionnellement à l'ordre social des galeries marchandes. Cette occupation alternative de certains recoins du centre commercial ne va pas sans heurts, notamment avec les clients ou les agents de sécurité.

5 L'approche interactionniste privilégiée ici nous permet de montrer en quoi le centre commercial fait l'objet d'une négociation de son usage, du moins d'un arrangement en situation entre plusieurs types d'acteurs. Ces procédés interactionnels de négociation nous renvoient directement à la question de la structuration de l'ordre social dans l'espace marchand.

6 Si les travaux de La Pradelle (1996; 2002), Poupard (2005) et Chevalier (2007) ont permis de montrer que les centres commerciaux (et autres espaces économiques urbains) ne se réduisent pas à leur réalité utilitaire et marchande - dans la mesure où ils permettent de cristalliser des formes de sociabilité qui s'opposent aux logiques économiques instituées -, ces derniers s'intéressent principalement à la clientèle ou encore aux jeunes (collégiens et lycéens) qui s'emparent aussi de ces lieux (LTMU, 2007). Pour notre part, il s'agit de prolonger leurs analyses en nous intéressant davantage à des personnes âgées qui s'ancrent au quotidien dans l'espace étudié et en détournent l'usage à des fins de sociabilité. En ce sens, nous nous démarquons également des recherches de Freitas (1996) et de Capron (1997) qui abordent plutôt le centre commercial dans ses dimensions architecturales, imaginaires ou politiques.

7 Après avoir mis en lumière les différentes pratiques par lesquelles les habitués s'approprient et détournent l'usage et le sens premier du centre commercial, nous 
étudierons les tactiques de négociation qui régulent la coprésence des différents acteurs dans le Centre.

\section{Les modalités d'appropriation de l'espace intérieur du centre commercial}

Figures 1 et 2

\section{REZ DE CHAUSSEE}

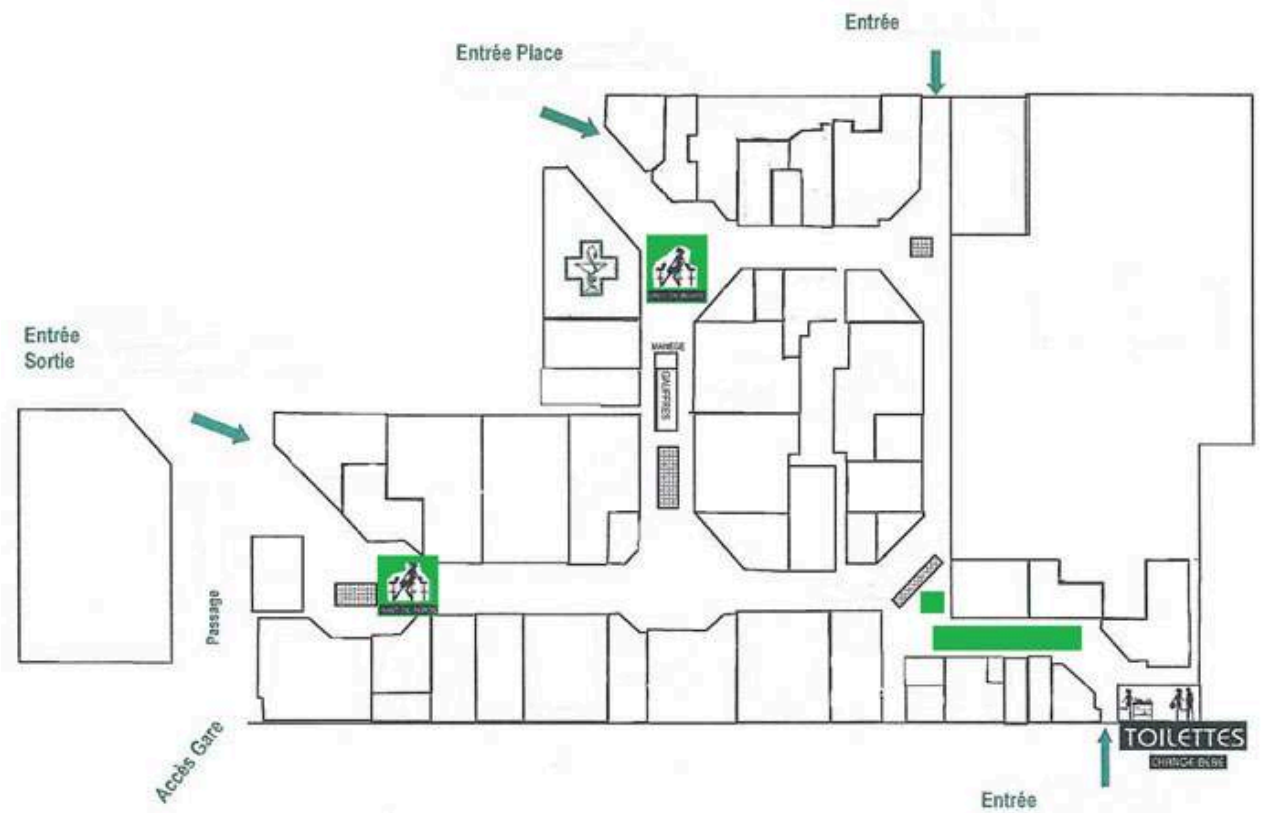

1er ETAGE

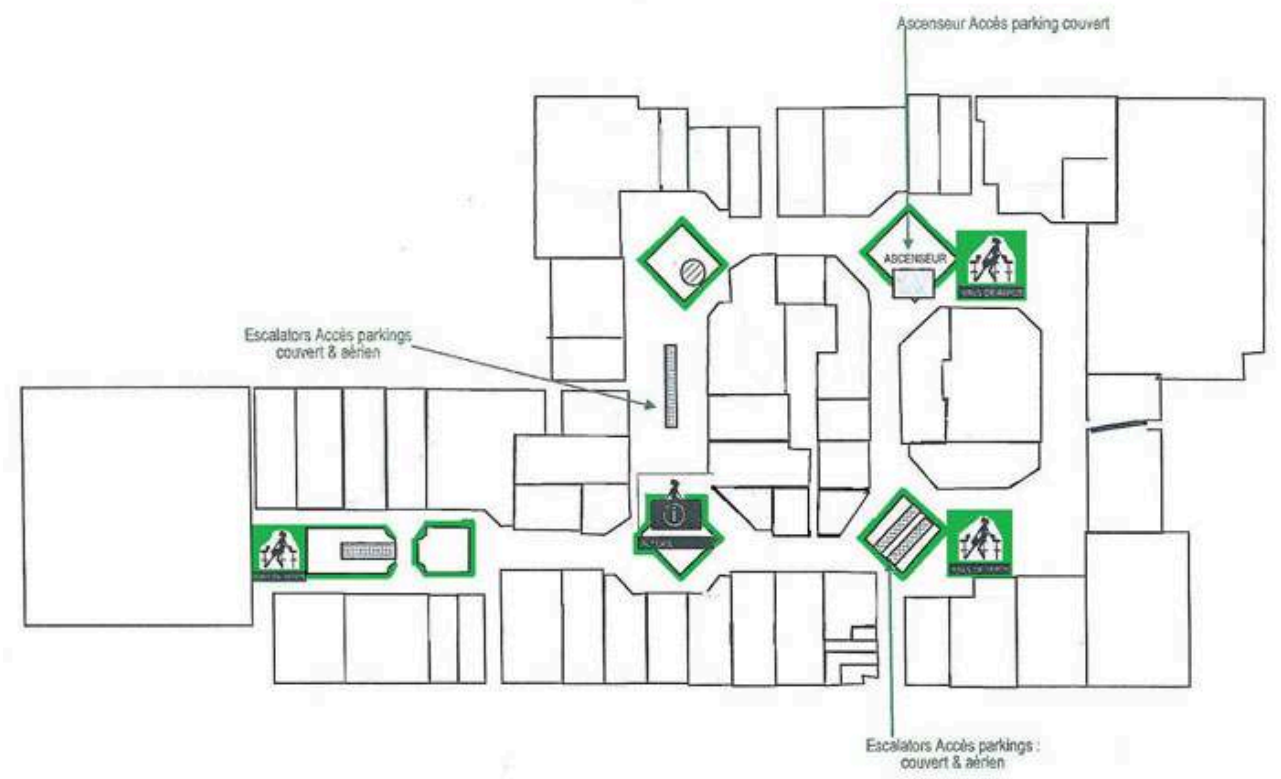

Légende : En vert, les micro-espaces appropriés par les habitués

Thibaut Besozzi ; source des plans : Centre Commercial. 


\section{Index des informateurs ${ }^{4}$}

- M. François : Homme, 78 ans, retraité de maçonnerie depuis 18 ans. Présent dans le Centre 4 à 6 fois par semaine, toujours à la même rambarde.

-M. Blanc : Homme, 78 ans, retraité du bâtiment, souvent assis isolément.

- M. David : Homme retraité, plus de 60 ans, présent quotidiennement dans le Centre, souvent assis aux aires du premier étage.

- M. Alain : Homme, 43 ans, chômeur, ancien comptable. Se rend au Centre 3 à 5 fois par semaine depuis qu'il est chômeur.

- Mme Gentil : Femme, retraitée, environ 80 ans. Quotidiennement présente dans le Centre, au sein de l'aire de la pharmacie.

- Mme Grey : Femme, retraitée, environ 80 ans. Quotidiennement présente dans le Centre, au sein de l'aire de la pharmacie.

- Mme Frach : Femme, retraitée, 91 ans. Quotidiennement présente dans le Centre.

- Mme Cournoit : Femme, retraitée, environ 70 ans. Quotidiennement présente dans le Centre, au sein de l'aire de la pharmacie.

- M. Pierre-Vincent : Homme, plus de 70 ans, souvent assis isolément.

- M. Bavard : Homme, 66 ans, retraité depuis 3 ans, ancien pompier. Plutôt en déplacement qu'assis.

-M. Chris : Homme, 62 ans, retraité de La Poste. Plutôt en déplacement qu'assis.

- M. Rick : Homme (schizophrène), plus de 50 ans, souvent assis (parfois endormi) dans les aires du premier étage.

- M. Joe : Homme, 80 ans, retraité de l'informatique. Assis quotidiennement dans plusieurs aires.

- M. Jacques : Homme (buveur), 59 ans, ancien brancardier. Présent quotidiennement dans le Centre.

-M. Mohamed : Homme, 43 ans, agent de sécurité du Centre depuis 12 ans. espaces sont constitués par des montants en bois, de la végétation et une démarcation au sol qui en signifient les limites ; ce sont autant d'éléments matériels et végétaux qui participent à la délimitation des micro-espaces d'immobilité et en font des « coins » qui s'opposent à l'injonction de circuler régissant l'usage des galeries ${ }^{5}$ (Poupard, 2005). Ces éléments matériels participent de l'autonomisation des normes informelles et de l'usage des aires.

11 L'usage conventionnel des micro-espaces d'immobilité se veut relativement rapide et utilitaire. Les clients s'assoient dans les aires de repos pendant des durées qui excèdent rarement une quinzaine de minutes : pour manger un sandwich, écrire un SMS, jeter un regard aux achats effectués ou simplement reposer des jambes que le « lèche-vitrines » fatigue. D'autres peuvent y calmer leur jeune enfant ou y attendre leur conjoint. Ce 
sont là les usages les plus récurrents au sein des aires de repos : nos entretiens avec le directeur du Centre aussi bien qu'avec les agents de sécurité confirment ces attentes normatives (Goffman, 2013) précisément respectées par les clients.

L'utilisation qui est faite du centre commercial par les habitués est tout à fait différente. Il est frappant de remarquer, lorsqu'on y prête attention, la présence quasiquotidienne de personnes âgées qui s'installent sur les fauteuils des aires de repos, ou qui s'accoudent aux rambardes, et ce pendant des temps relativement longs. Ils peuvent en effet passer plusieurs heures d'affilées à l'intérieur du Centre, à discuter en groupe, à flâner à la recherche d'un interlocuteur ou d'une place assise, ou encore assis seuls.

tit monde dans lequel s'inscrivent les personnes qui nous intéressent se structure d'abord par des procédés temporels d'appropriation de l'espace. Les habitués se démarque des clients par le fait qu'ils occupent les espaces intérieurs de manière plus fréquente - plusieurs fois par semaine, voire tous les jours - de façon plus régulière attestant d'habitudes hebdomadaires et quotidiennes - et surtout de manière plus longue - certains habitués restant pendant trois ou quatre heures au centre commercial, et ce chaque jour où ils s'y rendent. Cette régularité prend parfois une forme tellement rigide qu'elle n'est pas sans illustrer de véritables rites quotidiens. C'est par exemple le cas de $\mathrm{M}$. Alain qui donne rituellement son fauteuil à la même personne lorsqu'il s'en va, ou de $\mathrm{M}$. François qui rejoint «sa » rambarde d'élection à $15 \mathrm{~h} 30$ précises, pour la quitter à $17 \mathrm{~h}$. Ce qu'il faut souligner, c'est que le microcosme de sociabilité ne pourrait avoir de continuité sans cette structure temporelle (individuelle et collective) : elle est le soubassement nécessaire et indispensable à la stabilisation du phénomène d'appropriation observé.

Il existe par ailleurs une certaine organisation socio-spatiale de l'espace intérieur du Centre. Nous entendons par là que les habitués se distribuent silencieusement l'usage de l'espace intérieur en fonction de plusieurs critères que sont les horaires, le genre, l'appartenance culturelle ou encore le degré de marginalité. Suivant cette répartition spatiale informelle, les acteurs qui composent le microcosme en viennent à définir leur " place » à l'intérieur du Centre (Lussault, 2009).

15 Par exemple nous avons observé un groupe d'hommes âgés qui s'installe régulièrement à l'aire près de l'ascenseur - à l'étage - tandis qu'un groupe de femmes retraitées s'est approprié l'aire proche de la pharmacie - au rez-de-chaussée ${ }^{6}$. Si nous pouvons saisir d'un point de vue extérieur cette répartition, le plus important est de constater que les acteurs eux-mêmes ont conscience de cette organisation tacite. Les propos des femmes du rez-de-chaussée sont révélateurs : " Y'a plus assez d'endroits de rencontres vous savez... [...] nous on est ici, y'en a aussi là-haut des sièges, mais on y va pas, et eux viennent pas ici ». Ce à quoi répond cette critique d'un homme du groupe de l'étage : "L'autre vieille en bas, elle arrive à $14 h$ elle repart à $18 \mathrm{~h}$ !». On peut percevoir à travers ces exemples à la fois la connaissance que les habitués développent de leur espace et de leurs semblables, mais aussi l'animosité qui s'exerce parfois entre eux.

16 Cette distribution informelle de l'espace s'organise notamment en fonction des horaires. Il existe des habitués du matin, nombreux sont ceux de l'après-midi, et quelques-uns (les plus marginaux) profitent de la tranche horaire de $19 \mathrm{~h}$ à $20 \mathrm{~h} 30$ pour $s^{\prime}$ approprier les aires de repos ${ }^{7}$. Les vigiles sont alors beaucoup moins regardants quant à l'accessibilité aux galeries dans la mesure où les clients désertent le centre commercial après $19 \mathrm{~h}$. 
17 L'appartenance culturelle est un autre critère de cette organisation spatiale du groupediffus. Certains groupes de sociabilité sont composés de membres d'une même communauté qui se retrouvent à un endroit précis du Centre. C'est le cas du groupe d'italiens, du groupe de portugais, et de manière plus large des maghrébins ${ }^{8}$.

Enfin, c'est parfois le degré de précarité qui détermine l'usage des galeries intérieures. En effet, lors des périodes froides d'hiver, les agents de sécurité desserrent quelque peu leurs critères d'éviction et tolèrent certains marginaux plus visibles. Cela dit, la présence de personnes sans domicile fixe (SDF) et de mendiants semble se confiner dans un passage précis du centre commercial; celui qui mène vers l'arrière du bâtiment (en bas à droite sur le plan du rez-de-chaussée) et se compose des siègesronds les moins confortables parmi l'offre de mobilier urbain proposée par le Centre.

Par ailleurs, les habitués déplacent régulièrement les fauteuils des aires de repos pour en faire de petits salons de discussion imprévus. C'est notamment le cas des femmes habituées de l'aire de la phamarcie: Mme Cournoit, Mme Gentil, Mme Grey ou encore Mme Frach. Elles tournent les sièges les uns en face des autres ou en cercle et discutent collectivement, parfois pendant plusieurs heures. Ces procédés de transformation de l'espace participent éminemment du processus d'appropriation que nous souhaitons mettre en lumière. En effet, les habitués se saisissent de la possibilité d'arranger l'espace à leur manière. Nous avons ainsi pu observer jusqu'à une dizaine de personnes ${ }^{9}$ au sein d'une des aires de l'étage. Les clients ont une tendance nettement plus réduite au déplacement des fauteuils : ils s'y assoient le plus souvent sans les bouger ni même en changer l'orientation. Pour les habitués, l'importance de cette possibilité de modifier l'arrangement des micro-espaces appropriés s'exprime tant au niveau individuel qu'au niveau collectif. Certaines personnes s'en servent pour s'isoler spatialement, comme M. Pierre-Vincent ou M. Blanc qui tournent systématiquement leur fauteuil vers le couloir et s'écartent du centre de l'aire, tandis que d'autres les transforment en petits salons.

20 En outre, c'est dans les moments de sociabilité en groupe que l'appropriation spatiale est la plus forte et la plus visible. Nous parlons alors d'une mise en scène de la présence des habitués que leur gestuelle expansive et le volume de leurs paroles corroborent. Les aspects de cette mise en scène participent clairement et directement des pratiques d'appropriation observées. Les "marqueurs territoriaux » inventoriés par Goffman sont constitutifs de cette "représentation » - au sens théâtral - que jouent les acteurs qui nous intéressent (Goffman, 1973). Suivant cela, d'autres procédés subtils d'appropriation sont encore perceptibles. En effet, objets, corps et choses peuvent servir à délimiter l'espace d'intimité qui est approprié. De la sorte, ce sont essentiellement les corps qui font ici office de marqueurs, à savoir que les habitués s'appuient sur la présence de leurs semblables pour garder des places assises. Il nous a été donné d'observer une dame qui fit trois aller-retours entre l'aire de repos et le distributeur gratuit de tisane situé non loin, de manière à servir trois ou quatre hommes assis. Elle se justifiait en riant : «Ils ont peur de perdre leur place ».

21 L'appropriation spatiale se donne également à voir par d'autres comportements. À travers leurs postures corporelles et la connaissance qu'ils développent du lieu, les habitués se muent en quelque sorte en indigènes à leur aise dans le Centre. Ils peuvent transformer les micro-espaces comme nous l'avons vu, mais ont également une appréhension toute familière des différentes aires. Ainsi M. Alain nous dira qu'il préfère celle près de la pharmacie plutôt que l'aire proche de l'entrée ${ }^{10}$ « car il y a moins 
de passage ». Ainsi également de M. Joe qui n'apprécie guère le matériau de certains fauteuils situés à l'étage et sélectionne en conséquence les aires qu'il affectionne. Ce sont aussi leurs rapports corporels aux objets et à l'espace ainsi que leur appréhension sensorielle qui constituent cette familiarité à l'espace. Par exemple M. Alain ne s'assied pas sur tel fauteuil «parce qu'on sent le courant d'air de la porte ». Tandis qu'à l'instar de M. David, nombreux sont les habitués qui s'immobilisent avachis dans un fauteuil ou croisant ostensiblement les jambes, d'autres allant même jusqu'à y dormir. Par leur expression corporelle, les acteurs que nous avons observés donnent à voir la familiarité sensorielle qu'ils ont avec l'espace intérieur du centre commercial: cette aisance contraste avec les postures corporelles des clients. Ces derniers sont plus droits, plus discrets, ils mettent plus de distance dans leur rapport à l'espace, là où les habitués affichent une proximité, une certaine intimité à ce qui devient pour eux un lieu de vie. La "proxémie » est un outil d'analyse adéquat pour saisir ces diverses distances corporelles et spatiales (Hall, 1978). Toujours sur le plan sensoriel, d'autres habitués privilégient systématiquement telle ou telle visibilité : ils tournent leur fauteuil dans une direction qu'ils affectionnent en fonction de l'angle de vue qu'ils désirent avoir. C'est aussi un des intérêts que revêtent les rambardes: un groupe de Portugais s'accoudent par exemple régulièrement aux rambardes de l'étage; comme les Italiens déjà cités, ils observent le passage et les activités du rez-de-chaussée à partir de ces emplacements de choix.

Enfin, les personnes sur lesquels nous avons concentré nos observations attestent de leur ancrage dans le Centre à travers l'usage qu'ils font de certains services gratuits proposés dans les galeries marchandes. C'est-à-dire qu'ils ont une connaissance localisée des ressources qu'offre l'institution économique dont ils détournent l'usage. La consultation du journal en libre accès au point d'accueil est un exemple récurrent. Cet usage des services destinés à la clientèle ne se limite pas à cela. Nous pouvons par exemple y inclure l'utilisation des toilettes publiques entretenues aux frais du centre commercial. De plus, à l'aire près de l'ascenseur, un magasin propose de la tisane en libre-service : celle-ci est assez prisée par les habitués, particulièrement durant la période hivernale. Le responsable de la boutique proposant la boisson chaude nous le confirme : «On dirait qu'ils s'attendent entre eux. [...]. La tisane, ils en profitent bien (rire)». Quoi qu'il en soit, c'est l'ensemble du phénomène que nous pouvons inclure dans ces illustrations du détournement des ressources d'un tel espace. En effet, ici, tant la température climatisée que la musique d'ambiance ou la surveillance des vigiles sont des services du Centre qui profitent régulièrement aux acteurs que nous observons.

L'ensemble de ces pratiques clandestines, régulièrement observées, fait écho au sentiment d'appartenance à l'espace que développent les habitués; en ce sens, nous pouvons parler d'une appropriation spatiale à la fois pratique et mentale. C'est que les galeries marchandes fourmillent de "prises » (Joseph, 1997) qui sont effectivement saisies par les personnes âgées étudiées. Joseph définit ce terme comme suit: «Une prise, ou affordance, est une disponibilité pratique dans un contexte et pour une activité donnés ». En ce sens, les habitués s'emparent de l'espace intérieur du centre commercial et y investissent leur être corporel et subjectif. Ce n'est pas autre chose que veut dire M. François lorsqu'il désigne «sa » rambarde : «On a mis des racines ici ». En s'emparant de ces prises, les habitués s'inscrivent durablement et significativement dans ce qui devient subjectivement leur espace, leur place. À cet égard, les propos de M. Alain sont 
explicites: «Moi je n'ai pas de téléphone portable. Les gens qui veulent me voir viennent ici... c'est ici mon portable » (en désignant l'aire de repos).

Le microcosme de sociabilité créé par les habitués à partir de ces multiples procédés d'appropriation spatiale illustre avec précision la capacité d'adaptation des individus et des groupes sociaux à leur espace de vie quotidienne. Cela n'est pas sans faire écho à l'écologie urbaine de la tradition sociologique de Chicago. Nous attestons des arrangements spatiaux à travers lesquels différents groupes sociaux se distinguent et coexistent en ville (Joseph, Grafmeyer, 2004).

Nous avons appréhendé la micro-écologie urbaine qui se donne à voir dans le Centre à travers plusieurs éléments empiriques que sont: l'organisation tacite du groupe dans l'espace, la capacité de transformation des aires de repos, la mise en scène de la présence du groupe, la familiarité qui se développe avec le lieu ou encore le détournement effectif des ressources à disposition. En décrivant cette écologie localisée nous nous inscrivons directement dans la continuité des travaux de Poupard (2005).

\section{La négociation de l'appropriation spatiale du centre commercial}

Nous souhaitons désormais prendre en compte le fait que ce phénomène d'appropriation et de sociabilité ne va pas de soi. En effet, les acteurs étudiés se fondent tant bien que mal dans la réalité utilitaire du Centre et dans le monde plus conventionnel des clients. Leur présence et leur activité demeurent vulnérables parce qu'informelles et non-conventionnelles. Pour penser les modalités d'établissement de leur microcosme clandestin, il nous faut revenir sur la notion d'ordre social et tenter de voir comment se structure la réalité à l'intérieur du centre commercial. Loin de s'en tenir à ses aspects institués, formels et rationnalisés, le Centre implique aussi la réalité informelle et clandestine que "construisent» les habitués, au sens de Berger et Luckmann (2006).

Tout d'abord, il faut souligner la tension paradoxale qui traverse la conception matérielle et politique des centres commerciaux. Cette conception gestionnaire s'exprime par une volonté de la part des représentants de l'institution d'accueillir leur clientèle du mieux qu'ils le peuvent, c'est-à-dire avec un maximum de confort et de sérénité. Historiquement, une idéologie du bien-être du consommateur émerge progressivement sous la critique antérieure de l'utilitarisme trop criant de ces espaces économiques urbains (Berdet, 2013). Le directeur du Centre emploie à ce propos les termes de "parcours client » qu'aucun " obstacle » (matériel, visuel, olfactif, etc.) ne doit perturber. Les micro-espaces de repos s'inscrivent officiellement dans le cadre de cette idéologie du confort-client.

Mais le paradoxe apparaît dès lors que certains citadins (à l'image des habitués) sollicitent ces ressources de confort alors même qu'ils n'affichent que rarement des comportements marchands. Ces derniers sont alors non seulement inutiles aux objectifs de l'institution, mais également susceptibles de perturber l'activité commerciale organisée. Le directeur du Centre est explicite à ce sujet : « La société que je représente n'accepterait pas qu'il y ait des SDF qui soient présents dans le Centre. C'est un choix mesuré et économique. [...]. C'est un système euh... policier, quasiment quoi !». Le contrôle et l'exclusion éventuelle de ces personnes semblent donc être une volonté formelle de 
l'institution, mais les critères et les limites de cette autorité ne se laissent pas facilement définir.

Pour Goffman "un ordre social peut être défini comme la conséquence de tout ensemble de normes morales qui régule la façon dont les personnes poursuivent leurs objectifs» (2013). Fort de cette définition, nous pouvons observer le rapport entre l'ordre social des habitués et les autres ordres sociaux des espaces économiques (clients et professionnels) pour voir comment ces «mondes » coexistent et s'arrangent de leur présence mutuelle. La perspective adoptée interroge les moyens mis en œuvre par les habitués pour faire accepter leurs pratiques non-conventionnelles et informelles à l'intérieur du Centre une institution éminemment rationnalisée et contrôlée. Comment parviennent-ils à stabiliser ce phénomène d'appropriation et de détournement de l'espace?

Les procédés observés s'apparentent à des techniques d'adaptation et deviennent particulièrement intelligibles à partir du concept de négociation forgé par la seconde École de Chicago, et plus précisément par Strauss (1992). Il convient de ne pas le réduire à une négociation formelle, discursive et explicite, entre différents acteurs ayant des intérêts divergents. Ici, il s'agit bien plutôt d'une négociation implicite entre les trois ordres sociaux (clients, professionnels, habitués); arrangement tacite et rarement verbalisé qui s'exprime au niveau interactionnel. Ces arrangements nonofficiels se manifestent dans leur émergence en situation : ils ne sont pas perceptibles autrement que par l'approche empirique ici déployée. La recherche de Proth et Raybaud (2004) - à propos de la présence d'un petit groupe de SDF dans un aéroport fourmille d'exemples de négociations de l'usage alternatif de l'espace public que constitue l'aéroport.

31 Pour illustrer cela nous allons nous concentrer sur les modalités de contrôle qui régissent l'espace en question. Comment agissent les représentants de l'institution face à cette appropriation informelle? Quelles sont les "tactiques" (Certeau, 1990) déployées par les habitués pour faire accepter, tant bien que mal, leur activité déviante au sein de cet espace économique?

Lærégularité quasi-rituelle des procédés d'appropriation de l'espace mis en évidence dans la première partie est en elle-même un moyen de négociation du phénomène. Cette ritualisation renforce la visibilité du groupe et permet déjà une certaine emprise sur l'espace. Cela dit, une fois leur présence relativement tolérée, intégrée dans le cadre d'habitudes quotidiennes, les habitués ont encore à répondre aux « apparences normales » (Goffman, 1973) en dissimulant la durée de leur présence ou l'incongruité apparente de leurs comportements. Car si les vigiles ne peuvent exclure tous les habitués du seul fait qu'ils n'effectuent pas d'achat, ils peuvent néanmoins raccompagner à la porte certaines personnes dont le comportement déviant serait trop "visible » ou trop " gênant » pour la clientèle ou l'image du Centre. Un jeu interactionnel se met alors en place dont l'enjeu pour les habitués n'est autre que la survie de leur petit monde : ils développent différentes tactiques destinées à négocier leur présence avec la clientèle et avec les agents de sécurité.

1) Des « tactiques d'apitoiement ». Il s'agit là de parades verbales et non-verbales dont usent les habitués pour faire comprendre aux clients qu'ils revendiquent une place assise. Ce procédé interactionnel subtil s'observe régulièrement. Il consiste soit en une demande verbale prenant la forme générique suivante : «Je peux m'asseoir à votre place? »- en spécifiant ou non la norme de politesse, observable dans l'espace public, qui veut qu'on laisse les personnes âgées s'asseoir en priorité. Nous avons aussi pu voir ce type 
de demande: "Je suis malade, vous pouvez me laisser votre siège?». Mais le plus souvent, cette tactique d'apitoiement s'effectue de manière non-verbale. Elle consiste alors en un passage lent ou une stagnation au sein de l'aire de repos, en usant d'un regard insistant auprès des personnes assises afin qu'elles sentent la demande tacite. L'efficacité de cette tactique est avérée à tel point que les habitués n'ont souvent recours qu'à la parade non-verbale pour obtenir une place assise. La demande formelle pouvant suivre si cette première tentative n'aboutit pas.

34 2) Des «tactiques d'attente ». Il faut savoir que durant les heures de pointes (14h-18h) les places libres ne le restent rarement plus de quelques dizaines de secondes. Certains habitués peuvent attendre pendant plusieurs minutes qu'une place se libère. Ils dissimulent cette attente en maintenant les apparences normales comme ils le peuvent, tout en maximisant leur chance d'obtenir un fauteuil. Certains effectuent une ronde dans les galeries avec un net ralentissement de leur allure à l'abord d'une aire de repos (au cas où il $\mathrm{y}$ ait une opportunité de s'asseoir). D'autres stagnent aux vitrines près de l'aire convoitée afin d'être les premiers à s'emparer d'une place si celle-ci se libère, tout en gardant l'attitude d'un client qui repère des marchandises.

3) Des «tactiques d'effacement ». Certains habitués cherchent à réduire leur visibilité aux yeux des vigiles en s'asseyant successivement dans plusieurs aires de repos au lieu de rester toute l'après-midi dans le même fauteuil. Ils savent que leur présence est déviante et cherchent à se fondre dans le décor. De la sorte, ils peuvent rester plus longtemps à l'intérieur des galeries marchandes : soit qu'ils s'installent par tranche de demi-heure dans différents micro-espaces de repos; soit qu'ils restent debout, circulant d'un groupe à l'autre, à l'instar de M. Bavard ou M. Chris.

36 4) Des «tactiques de subversion ». Quelques habitués dorment au sein des aires de repos - ce qui est interdit et contrôlé par les agents de sécurité - en portant des lunettes de soleil pour cacher leurs yeux fermés. Ils savent que les vigiles sont susceptibles de venir les réveiller s'ils se reposent au point de dormir. D'autres, parmi les plus indigents, comme M. Jacques, s'arrangent pour quémander un peu d'argent à l'intérieur des galeries marchandes - ce qui est également interdit; mais ce dernier s'adresse uniquement aux habitués qu'il connaît afin de maintenir la discrétion.

Il faut considérer les marges de manœuvre dont disposent les vigiles dans leur application des règles. En effet, les agents de sécurité ferment parfois les yeux sur ces activités clandestines. Cela se décide en fonction de l'affluence dans les galeries, mais aussi en fonction des habitués eux-mêmes que les vigiles connaissent parfois personnellement. Ainsi, M. Mohamed qui est agent de sécurité du centre commercial depuis une douzaine d'années nous explique qu'il laisse M. Rick s'installer durablement et dormir dans les fauteuils car «il est schizophrène, mais il prend ses médocs... des fois ça l'assomme ». De la sorte, quelques habitués peuvent profiter d'une certaine immunité, bien que celle-ci demeure relative. Comme nous l'avons dit, les vigiles assouplissent leur contrôle vis-à-vis des citadins les plus indigents pendant les périodes froides de l'hiver. Ces derniers sont alors confinés dans le passage aux sièges-ronds « où ils dérangent moins".

Ce qu'il convient finalement de souligner, c'est que les habitués font montre d'une certaine réflexivité pour négocier leur présence auprès des usagers conventionnels du Centre et auprès des agents de contrôle des galeries. Ils déploient différentes pratiques plus ou moins ingénieuses afin de continuer à s'inscrire individuellement et collectivement à l'intérieur des galeries marchandes du Centre. 


\section{Conclusion}

Après avoir mis en évidence les multiples procédés spatiaux par lesquels s'instaure un véritable microcosme clandestin à l'intérieur du Centre, nous avons souligné les rapports de négociation qui permettent aux membres de ce microcosme d'assurer la continuité de leur petit monde, et ce, malgré le caractère informel et déviant de leurs pratiques quotidiennes. Nous inscrivant ainsi dans la continuité de certaines recherches déjà menées sur les usages sociaux des centres commerciaux (Freitas 1996 ; Poupard, 2005; Chevalier, 2007), nous proposons une optique microsociologique interactionniste qui met en évidence les pratiques socio-spatiales de certains acteurs et leurs procédés d'accommodation aux conditions institutionnelles préétablies par le Centre.

- Finalement, les acteurs étudiés s'arrangent de l'espace à partir des connaissances qu'ils ont des règles formelles et de leur malléabilité sur le plan informel, tandis que les agents de sécurité tentent de faire appliquer ces règles en se ménageant une marge d'interprétation. Ainsi, nous pouvons conclure que la réalité sociale propre au centre commercial n'est ni réductible à «l'ordre institutionnel ", ni indépendante de ses attentes rationalisées et formalisées (Céfaï, Perreau, 2013). C'est-à-dire que la réalité empirique du Centre ne se limite pas aux normes et usages institués. Au contraire, l'ordre social localisé se met en place et s'actualise dans la tension - qui se donne à voir au niveau de «l'ordre de l'interaction» (Goffman, Winkin, 1988) - entre les attentes conventionnelles et les pratiques effectives. Cette tension est inhérente à ce qu'il convient de nommer le processus de structuration de l'ordre social contextualisé. Loin de se réduire aux prescriptions formelles et aux programmations conçues institutionnellement, la réalité sociale s'ordonne autour des interstices dont se saisissent les acteurs - à partir des marges de liberté qui sont les leurs - pour interpréter, s'adapter et modifier la réalité institutionnelle. L'ordre social contextualisé émerge alors de l'imbrication des logiques institutionnelles formelles et des logiques interactionnelles informelles : entre aménagements et vécus quotidiens.

\section{BIBLIOGRAPHY}

Augé M., 1992, Non-lieux. Introduction à une anthropologie de la surmodernité, Paris, Seuil, 150 p.

Berdet M., 2013, Fantasmagories du capital. L'invention de la ville-marchandise, Paris, La Découverte, $265 \mathrm{p}$.

Berger P., Luckmann T., 2006, La construction sociale de la réalité, Paris, Armand Colin, 357 p.

Caillé A., 1998, « Don et association », La Revue du MAUSS semestrielle, n¹1, p. 75-83.

Capron G., 1997, « Les centres commerciaux à Buenos Aires. Les nouveaux espaces publics de la ville de la fin du XXè siècle », Les Annales de La Recherche Urbaine, nº78, p. 55-63. 
Céfaï D., Perreau L. (dir.), 2013, Erving Goffman et l'ordre de l'interaction, Paris, CURAPP, 466 p.

Chevalier S., 2007, « "Shopping" à la française: approvisionnement alimentaire et sociabilité », Horiz. antropol. [en ligne], vol.13, n.28, p. 65-86.

Chivallon C., Marme N., Prost D., 1997, « Artefact de lieu et urbanité. Le centre commercial interrogé. », Les Annales de la Recherche Urbaine, n78, p. 28-37.

Chombart de Lauwe P.-H., 1963, Des hommes et des villes, Paris, Éditions Payot, 267 p.

De Certeau M., 1990, L'invention du quotidien, Tome 1 : Arts de faire, Paris, Gallimard, 350 p.

De La Pradelle M., 1996, Les vendredis de Carpentras. Faire son marché en Provence ou ailleurs, Paris, Fayard, $374 \mathrm{p}$.

De La Pradelle M., 2002, « La fabrication de la ville au quotidien. Espaces marchands, espaces festifs et formes de citadinité » in Bajolet É., Mattéi M.-F., et Rennes J.-M. (éds), Quatre années de recherche urbain 2001-2004, vol.1, Tours : Presses Universitaires François-Rabelais, p. 147-154.

Ferreira Freitas R., 1996, Centres commerciaux : îles urbaines de la post-modernité, Paris, L'Harmattan, $142 \mathrm{p}$.

Goffman E., 1973, La mise en scène de la vie quotidienne, Tome 2 : les relations en public, Paris, Éditions de Minuit, $372 \mathrm{p}$.

Goffman E., Winkin Y., 1988, Les moments et leurs hommes, Paris, Seuil/Minuit, 306 p.

Goffman E., 2013, Comment se conduire dans les lieux publics, Paris, Economica, 253 p.

Hall E.T., 1968, La dimension cachée, Paris, Éditions du Seuil, 256 p.

Joseph I., 1997, « Prises, réserves, épreuves », Communications, n65, p.131-142.

Joseph I., Grafmeyer Y. (dir.), 2004, L'école de Chicago. Naissance de l'écologie urbaine, Paris, Flammarion, $378 \mathrm{p}$.

Laboratoire Théories des Mutations Urbaines, 2007, Jeunes métropolitains aux Halles, CEME/LTMU/ Ville de Paris. 67 p.

Lussault M., 2009, De la lutte des classes à la lutte des places, Paris, Grasset, 220 p.

Poupard J.-M., 2005, Les centres commerciaux. De nouveaux lieux de socialité dans le paysage urbain, Paris, L'Harmattan, $170 \mathrm{p}$.

Proth B., Raybaud V., 2004, « Une famille de SDF recomposée à l'aéroport », ethnographiques.org, $\mathrm{n}^{\circ} 6$, [en ligne].

Strauss A., 1992, La trame de la négociation. Sociologie qualitative et interactionniste, Paris, L'Harmattan, $311 \mathrm{p}$.

Weber M., 1992, Essais sur la théorie de la science, Paris, Pocket, 478 p.

\section{NOTES}

1. C'est le nom que nous lui donnerons. Nous l'appellerons parfois « le Centre ».

2. C'est le nom donné par le Centre aux espaces de détente attenants aux galeries marchandes. Nous les appellerons aussi « micro-espaces d'immobilité ».

3. Il s'agit de catégories idéales-typiques (Weber, 1992) construites par le chercheur. Elles simplifient en fait une réalité plus complexe et moins figée : rien n'empêche les clients de s'assoir longuement dans les aires, tandis que les habitués effectuent aussi parfois des actes marchands. 
Par précaution, nous devrions maintenir systématiquement l'usage des guillemets, ce que nous éviterons pour faciliter la lecture du texte.

4. Pour préserver l'anonymat de nos informateurs, il s'agit là de noms d'emprunt.

5. En effet, il est formellement interdit de s'assoir en dehors de ces aires de détente, c'est-à-dire sur le sol des couloirs.

6. Sur ce point, il faut d'ailleurs noter que la majorité des habitués du Centre sont des hommes.

7. Si le Centre clôt ses portes à $20 \mathrm{~h} 30$ chaque jour de la semaine, les boutiques intérieures ferment à 19h, hormis le supermarché qui reste ouvert jusqu'à 20h30. Il y a moins de « concurrence » pour l'usage des aires à ce moment de la journée.

8. En effet, ces derniers sont plus nombreux relativement aux italiens ou aux portugais, c'est pourquoi ils ne composent pas un groupe, mais plusieurs petits groupes qui peuvent se répartir dans différentes aires de repos.

9. Dont certaines restaient debout compte tenu du nombre restreint de places assises : de 5 à 7 places suivant les aires.

10. À gauche sur le plan du rez-de-chaussée.

\section{ABSTRACTS}

Based on the case of a shopping center in the city of Nancy, in which a phenomenon of appropriation of the inside space by groups of essentially elderly retired, we try to understand how the actors use the spatial organization proposed by the management of the commercial center and negotiate this spatial appropriation. From ethnographical data eminently from context, we suggest widening the reflection by showing that the urban social order emerges through a tension between the arrangements of the commercial space (which think about sociability) and the real-life experience of the same spaces, which gives evidence of the accommodation which make some special actors.

Cet article propose l'étude ethnographique d'un centre commercial situé en centre-ville de Nancy, dans lequel s'est mis en place, au quotidien, un phénomène d'appropriation de l'espace intérieur par des groupes de personnes âgées essentiellement retraitées. Ce sont surtout les petits espaces de détente qui sont utilisés voire privatisés par ces personnes. Nous cherchons à montrer comment ces acteurs s'accommodent de l'organisation spatiale proposée par la direction du centre commercial et comment ils négocient cette appropriation. À partir des données empiriques recueillies, nous proposons d'élargir la réflexion en montrant que l'ordre social urbain émerge plus généralement d'une tension entre la conception institutionnelle (formelle) de l'espace commercial et le vécu pratique (informel) de ces mêmes espaces.

Wir haben eine ethnographische Studie eines inmitten des Stadtzentrums von Nancy gelegenen Einkaufszentrums durchgeführt, in dem sich Gruppe von alten Leuten (meistens Rentnern) den Innenraum aneignen. Dieses Phänomen betrifft besonders kleine Aufenthaltsräume, die genutzt und sogar angeeignet werden. Wir gehen der Frage nach, wie sich die Akteure der von der Zentrumsleitung vorgeschlagten raümlichen Einrichtung anpassen und, wie sie diese räumliche Aneignung verhandeln. Aus empirischen Daten, möchten wir die Überlegung erweitern und beweisen, dass die gesellschaftliche Ordnung der Stadt aus einer Spannung zwischen der 
institutionnellen Anschauung des Einkaufsraums und der alltäglichen Nutzung dieses Raums herauskommt.

INDEX

Schlüsselwörter: alltägliche Nutzung, Einrichtung, raumliche Aneignung, Verhandlung Mots-clés: appropriation spatiale, conception de l'espace, négociation, vécu de l'espace Keywords: arrangements of space, negotiation, real-life experiences, spatial appropriation

\section{AUTHOR}

\section{THIBAUT BESOZZI}

Doctorant en sociologie - Université Paris-Dauphine, IRISSO - Université de Lorraine, 2L2S thibaut.besozzi@univ-lorraine.fr 